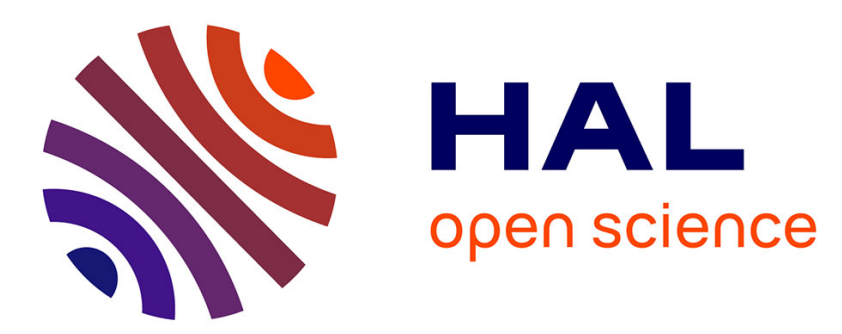

\title{
Microscopic theory of spin transport at the interface between a superconductor and a ferromagnetic insulator
}

T. Kato, Y. Ohnuma, M. Matsuo, Jérôme Rech, Thibaut Jonckheere, T. Martin

\section{- To cite this version:}

T. Kato, Y. Ohnuma, M. Matsuo, Jérôme Rech, Thibaut Jonckheere, et al.. Microscopic theory of spin transport at the interface between a superconductor and a ferromagnetic insulator. Physical Review B: Condensed Matter and Materials Physics (1998-2015), 2019, 99 (14), pp.144411. 10.1103/PhysRevB.99.144411 . hal-02196803

\section{HAL Id: hal-02196803 https://hal.science/hal-02196803}

Submitted on 29 Jul 2019

HAL is a multi-disciplinary open access archive for the deposit and dissemination of scientific research documents, whether they are published or not. The documents may come from teaching and research institutions in France or abroad, or from public or private research centers.
L'archive ouverte pluridisciplinaire HAL, est destinée au dépôt et à la diffusion de documents scientifiques de niveau recherche, publiés ou non, émanant des établissements d'enseignement et de recherche français ou étrangers, des laboratoires publics ou privés. 


\title{
Microscopic theory of spin transport at the interface between a superconductor and a ferromagnetic insulator
}

\author{
T. Kato, ${ }^{1}$ Y. Ohnuma, ${ }^{2}$ M. Matsuo, ${ }^{2}$ J. Rech,${ }^{3}$ T. Jonckheere, ${ }^{3}$ and T. Martin ${ }^{3}$ \\ ${ }^{1}$ Institute for Solid State Physics, The University of Tokyo, Kashiwa 277-8581, Japan \\ ${ }^{2}$ Kavli Institute for Theoretical Sciences, University of Chinese Academy of Sciences, Beijing 100190, China \\ ${ }^{3}$ Aix Marseille Univ, Université de Toulon, CNRS, CPT, Marseille, France
}

(Received 8 January 2019; revised manuscript received 28 March 2019; published 15 April 2019)

\begin{abstract}
We theoretically investigate spin transport at the interface between a ferromagnetic insulator (FI) and a superconductor (SC). Considering a simple FI-SC interface model, we derive formulas for the spin current and spin-current noise induced by microwave irradiation (spin pumping) or the temperature gradient (the spin Seebeck effect). We show how the superconducting coherence factor affects the temperature dependence of the spin current. We also calculate the spin-current noise in thermal equilibrium and in nonequilibrium states induced by the spin pumping and compare them quantitatively for an yttrium iron garnet- $\mathrm{NbN}$ interface.
\end{abstract}

DOI: 10.1103/PhysRevB.99.144411

\section{INTRODUCTION}

Spin transport in hybrid systems composed of superconductors (SCs) and ferromagnetic metals has been investigated for a long time [1-4]. In a superconductor, charge and spin imbalances may have different characteristic length scales due to spin-charge separation [5-7]. The interplay between superconductivity and magnetism also offers the potential for novel spintronic devices, in which fast logic operation can be performed with minimum Joule heating [8]. One of the key ingredients there is the injection of spin-polarized carriers into SCs [7,9-12]. For conventional $s$-wave superconductors, spin injection is suppressed by opening a superconducting gap in the electronic spectrum. Thermally excited quasiparticles in $\mathrm{SC}$, however, can carry a spin current over long distances, as spin excitations in SCs have long lifetimes [13-18].

There are several techniques for spin injection into SCs. Recently, it has been realized by taking advantage of the spin Seebeck effect (SSE) induced by a temperature gradient [19-25] or by applying a spin-pumping (SP) protocol using ferromagnetic resonance (FMR) under microwave irradiation [26-30]. The latter technique has successfully been used in experiments to realize spin injection from ferromagnetic metals into a SC [31-33]. These recent advances indicate a new path for spin injection into a wide class of SC materials. Remarkably, spin-current injection from a ferromagnetic insulator (FI) into a superconductor has also been performed recently [34], as revealed by the inverse spin Hall effect (ISHE) [35-37]. This last study opens up possible applications for novel superconducting spintronic devices using FI.

In contrast to progress in experiments, the spin current at the FI-SC interface has been studied theoretically, to our knowledge, only by Inoue et al. [38]. They formulated the spin-pumping signal in terms of the local spin susceptibility of the SC and showed that the signal is peaked below the transition temperature due to the coherence factor in the BCS theory. In order to calculate the local spin susceptibility of the SC, they employed the Abrikosov-Gor'kov theory for dirty
SCs taking spin diffusion into account [39-41]. The dynamic spin susceptibility thus obtained is, however, correct only for small wave numbers, whereas the local spin susceptibility, which involves all wave numbers, is dominated by the large wave number contribution [42] (for details, see Appendix A). Therefore, although their discovery of the coherence peak in spin transport is remarkable, their theory is expected to be insufficient for a quantitative description of the spin-current generation.

In this paper, we consider a bilayer system composed of an $s$-wave singlet SC and a FI, as shown in Fig. 1. We formulate the spin current at the interface and study its temperature dependence above and below the superconducting transition temperature. We also discuss the noise power of the pure spin current following the theory developed by three of the present authors and one collaborator [43] and estimate it using the experimental parameters for the yttrium iron garnet(YIG) $\mathrm{NbN}$ interface $[29,34]$.

This paper is organized as follows. We introduce the model for the FI-SC interface in Sec. II and derive dynamic spin susceptibilities in Sec. III. By using a second-order perturbative expansion with respect to the interface exchange coupling, we calculate the spin current and the spin-current noise in Secs. IV and V, respectively. It should be stressed that we evaluate the spin current just at the FI-SC interface. For experimental detection, one needs a nanostructure for converting the spin current into an electronic response, a mechanism which depends, in general, on details of spin relaxation in the superconductor. We briefly discuss such a possible experimental setup for detecting the spin current in Sec. VI. Finally, we summarize our results in Sec. VII. Detailed discussions of the impurity effect and the spin susceptibility of the SC are given in Appendixes A and B, respectively.

\section{MODEL}

The system Hamiltonian is given by $H=H_{\mathrm{SC}}+H_{\mathrm{FI}}+$ $H_{\mathrm{ex}}$. The first term, $H_{\mathrm{SC}}$, describes a bulk SC and is given by 


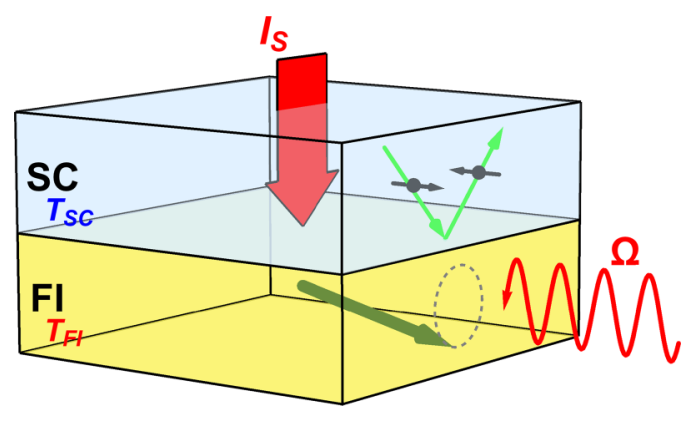

FIG. 1. Schematic picture of the FI-SC bilayer system. A spin current $I_{S}$ is generated in the SC by spin pumping using an external microwave irradiation or by the spin Seebeck effect induced by a temperature gradient $\left(T_{\mathrm{FI}} \neq T_{\mathrm{SC}}\right)$. The large green arrow in the FI illustrates the magnetization, which can precess due to the applied microwave at frequency $\Omega$. The arrows in the SC show an example of electron reflection at the interface, with a spin flip due to the exchange interaction.

the mean-field Hamiltonian

$$
H_{\mathrm{SC}}=\sum_{k}\left(c_{k \uparrow}^{\dagger}, c_{-k \downarrow}\right)\left(\begin{array}{cc}
\xi_{k} & \Delta \\
\Delta & -\xi_{k}
\end{array}\right)\left(\begin{array}{c}
c_{k \uparrow} \\
c_{-k \downarrow}^{\dagger}
\end{array}\right),
$$

where $c_{k \sigma}\left(c_{k \sigma}^{\dagger}\right)$ is the annihilation (creation) operator of the electrons in the superconductors and $\xi_{k}$ is the energy of conduction electrons measured from the chemical potential. The order parameter of the SC $\Delta$ is determined by the gap equation

$$
\ln \left(\frac{T}{T_{\mathrm{c}}}\right) \Delta=2 \pi T \sum_{\varepsilon_{n}>0}\left(\frac{\Delta}{\sqrt{\varepsilon_{n}^{2}+\Delta^{2}}}-\frac{\Delta}{\epsilon_{n}}\right),
$$

where $\varepsilon_{n}=(2 n+1) \pi T$ is the Matsubara frequency and $T_{\mathrm{c}}$ is the SC transition temperature [38].

The second term $H_{\mathrm{FI}}$ describes a bulk FI and is given by the Heisenberg model

$$
\begin{aligned}
H_{\mathrm{FI}}= & \sum_{\langle i, j\rangle} J_{i j} \boldsymbol{S}_{i} \cdot \boldsymbol{S}_{j}-\hbar \gamma h_{\mathrm{dc}} \sum_{i} S_{i}^{z} \\
& -\frac{\hbar \gamma h_{\mathrm{ac}}}{2} \sum_{i}\left(e^{-i \Omega t} S_{i}^{-}+e^{i \Omega t} S_{i}^{+}\right),
\end{aligned}
$$

where $S_{i}$ is the localized spin at site $i$ in the FI, $J_{i j}$ is the exchange interaction, $h_{\mathrm{dc}}$ is a static magnetic field, $h_{\mathrm{ac}}$ and $\Omega$ are the amplitude and frequency of the applied microwave radiation, respectively, and $\gamma$ is the gyromagnetic ratio. Using the Holstein-Primakoff transformation [44] and employing the spin-wave approximation $\left(S_{j}^{z}=S_{0}-b_{j}^{\dagger} b_{j}, S_{j}^{+} \simeq\left(2 S_{0}\right)^{1 / 2} b_{j}\right)$, the Hamiltonian of the FI is rewritten as

$$
\begin{aligned}
H_{\mathrm{FI}} \simeq \text { const } & +\sum_{\boldsymbol{k}} \hbar \omega_{\boldsymbol{k}} b_{\boldsymbol{k}}^{\dagger} b_{\boldsymbol{k}} \\
& -\frac{\hbar \gamma h_{\mathrm{ac}}}{2} \sqrt{2 S_{0} N_{\mathrm{F}}}\left(e^{-i \Omega t} b_{\boldsymbol{k}=\mathbf{0}}^{\dagger}+e^{i \Omega t} b_{\boldsymbol{k}=\mathbf{0}}\right),
\end{aligned}
$$

where $\hbar \omega_{k}$ is the magnon dispersion, $b_{k}$ is the Fourier transform of $b_{j}, S_{0}$ is the magnitude of the localized spin, and $N_{\mathrm{F}}$ is the number of spins in the FI. For simplicity, we assume a parabolic dispersion $\hbar \omega_{k}=D k^{2}+E_{0}$, where $E_{0}=\hbar \gamma h_{\mathrm{dc}}$ is the Zeeman energy.

The last term in the system Hamiltonian, $H_{\mathrm{ex}}$, describes the exchange coupling at the interface. In this paper, we employ a simple model using the following tunneling Hamiltonian for spins:

$$
H_{\mathrm{ex}}=\sum_{k, q}\left[\mathcal{T}_{k, q} S_{k}^{+} s_{q}^{-}+\text {H.c. }\right],
$$

where $\mathcal{T}_{\boldsymbol{k}, \boldsymbol{q}}$ is the tunneling amplitude, $S_{\boldsymbol{k}}^{+}=\left(2 S_{0}\right)^{1 / 2} b_{\boldsymbol{k}}$, and $s_{\boldsymbol{q}}^{-}$is an operator defined as

$$
s_{q}^{-}:=\sum_{k} c_{k \downarrow}^{\dagger} c_{k+q \uparrow} .
$$

In what follows, we study the spin transport by considering a second-order perturbative expansion with respect to $H_{\mathrm{ex}}$.

\section{DYNAMIC SPIN SUSCEPTIBILITY}

In this section, we summarize the results for the dynamic spin susceptibilities for the unperturbed system, i.e., the decoupled FI and SC, which are later used in the second-order perturbation calculation of the spin current and spin-current noise.

\section{A. Retarded component}

We define the retarded components of the spin susceptibility of the SC and the magnon propagator in the FI as

$$
\begin{gathered}
\chi^{R}(\boldsymbol{q}, t):=i\left(\hbar N_{\mathrm{S}}\right)^{-1} \theta(t)\left\langle\left[s_{\boldsymbol{q}}^{+}(t), s_{\boldsymbol{q}}^{-}(0)\right]\right\rangle, \\
G^{R}(\boldsymbol{k}, t):=-i \hbar^{-1} \theta(t)\left\langle\left[S_{\boldsymbol{k}}^{+}(t), S_{\boldsymbol{k}}^{-}(0)\right]\right\rangle,
\end{gathered}
$$

where $N_{\mathrm{S}}$ is the number of unit cells in the SC. Their Fourier transformations are defined as

$$
\begin{aligned}
\chi^{R}(\boldsymbol{q}, \omega) & :=\int_{-\infty}^{\infty} d t e^{i \omega t} \chi^{R}(\boldsymbol{q}, t), \\
G^{R}(\boldsymbol{k}, \omega) & :=\int_{-\infty}^{\infty} d t e^{i \omega t} G^{R}(\boldsymbol{k}, t) .
\end{aligned}
$$

We first consider the magnon propagator of the FI. By using the Holstein-Primakoff transformation [44] and employing the spin-wave approximation $\left[S_{k}^{+} \simeq\left(2 S_{0}\right)^{1 / 2} b_{k}\right]$, the magnon propagator in the FI is calculated in the absence of the external field $\left(h_{\mathrm{ac}}=0\right)$ as

$$
G^{R}(\boldsymbol{k}, \omega)=\frac{2 S_{0} / \hbar}{\omega-\omega_{\boldsymbol{k}}+i \alpha \omega},
$$

where we have introduced the phenomenological dimensionless damping parameter $\alpha$, which originates from the Gilbert damping.

Next, we consider the dynamic spin susceptibility of the $\mathrm{SC}$ in the BCS theory. We define the local spin susceptibility as

$$
\chi_{\mathrm{loc}}^{R}(\omega):=\frac{1}{N_{\mathrm{S}}} \sum_{q} \chi^{R}(\boldsymbol{q}, \omega)
$$


In the BCS theory, the local spin susceptibility is calculated as [45]

$$
\begin{aligned}
\operatorname{Im} \chi_{\mathrm{loc}}^{R}(\omega)= & -\pi N\left(\epsilon_{\mathrm{F}}\right)^{2} \int d E\left[1+\frac{\Delta^{2}}{E(E+\hbar \omega)}\right] \\
& \times[f(E+\hbar \omega)-f(E)] D(E) D(E+\hbar \omega), \\
& D(E)=\frac{|E|}{\sqrt{E^{2}-\Delta^{2}}} \theta\left(E^{2}-\Delta^{2}\right),
\end{aligned}
$$

where $N\left(\epsilon_{\mathrm{F}}\right)$ is the density of states per spin and per unit cell, $f(E)=\left(e^{E / k_{\mathrm{B}} T}+1\right)^{-1}$ is the Fermi distribution function, $D(E)$ is the (normalized) density of states of quasiparticles, and $\theta(x)$ is the Heaviside step function (for a detailed derivation, see Appendix B). We note that the factor $\left[1+\Delta^{2} / E(E+\hbar \omega)\right]$ in Eq. (13) is the so-called coherence factor, which produces singular behavior near the transition temperature [45]. For the normal metal $(\Delta=0)$, the local spin susceptibility becomes

$$
\operatorname{Im} \chi_{\mathrm{loc}, \Delta=0}^{R}(\omega)=\pi N\left(\epsilon_{\mathrm{F}}\right)^{2} \hbar \omega .
$$

\section{B. Lesser component}

We define the lesser components of the spin susceptibilities for bulk SC and FI as

$$
\begin{aligned}
\chi^{<}(\boldsymbol{q}, t) & :=i\left(\hbar N_{\mathrm{S}}\right)^{-1}\left\langle s_{\boldsymbol{q}}^{-}(0) s_{\boldsymbol{q}}^{+}(t)\right\rangle, \\
G^{<}(\boldsymbol{k}, t) & :=-i \hbar^{-1}\left\langle S_{\boldsymbol{k}}^{-}(0) S_{\boldsymbol{k}}^{+}(t)\right\rangle .
\end{aligned}
$$

Their Fourier transformations are defined as

$$
\begin{aligned}
& \chi^{<}(\boldsymbol{q}, \omega)=\int_{-\infty}^{\infty} d t e^{i \omega t} \chi^{<}(\boldsymbol{q}, t), \\
& G^{<}(\boldsymbol{k}, \omega)=\int_{-\infty}^{\infty} d t e^{i \omega t} G^{<}(\boldsymbol{k}, t) .
\end{aligned}
$$

The lesser components include the information on the distribution function; we define the distribution functions as

$$
\begin{aligned}
f^{\mathrm{SC}}(\boldsymbol{q}, \omega) & :=\chi^{<}(\boldsymbol{q}, \omega) /(2 i) \operatorname{Im} \chi^{R}(\boldsymbol{q}, \omega), \\
f^{\mathrm{FI}}(\boldsymbol{k}, \omega) & :=G^{<}(\boldsymbol{k}, \omega) /(2 i) \operatorname{Im} G^{R}(\boldsymbol{k}, \omega) .
\end{aligned}
$$

In the setup of the SP, the SC is in equilibrium with the temperature $T$, whereas magnons in FI are excited by the external microwave irradiation. We split the Hamiltonian of the FI as $H_{\mathrm{FI}}=H_{0}+V$, where

$$
\begin{gathered}
H_{0}=\sum_{k} \hbar \omega_{\boldsymbol{k}} b_{\boldsymbol{k}}^{\dagger} b_{\boldsymbol{k}}, \\
V=-h_{\mathrm{ac}}^{+}(t) b_{0}^{\dagger}-h_{\mathrm{ac}}^{-}(t) b_{0}, \\
h_{\mathrm{ac}}^{ \pm}(t)=\frac{\hbar \gamma h_{\mathrm{ac}}}{2} \sqrt{2 S_{0} N_{F}} e^{\mp i \Omega t} .
\end{gathered}
$$

While the perturbation $V$ does not change the retarded component of the dynamic spin susceptibility of FI, it does modify the lesser component. The second-order perturbation with respect to $V$ gives the correction:

$$
\begin{array}{r}
\delta G^{<}(\boldsymbol{k}, \omega)=G_{0}^{R}(\boldsymbol{k}, \omega) \Sigma(\boldsymbol{k}, \omega) G_{0}^{A}(\boldsymbol{k}, \omega), \\
\Sigma(\boldsymbol{k}, \omega)=\delta_{\boldsymbol{k}, \mathbf{0}} \int d t\left(-i \hbar^{-1}\right)\left\langle h_{\mathrm{ac}}^{-}(t) h_{\mathrm{ac}}^{+}(0)\right\rangle e^{i \omega t},
\end{array}
$$

where $G_{0}^{R}(\boldsymbol{k}, \omega)$ is the unperturbed spin susceptibility of FI. One can then straightforwardly obtain

$$
\begin{aligned}
\delta f^{\mathrm{FI}}(\boldsymbol{k}, \omega) & =\delta G^{<}(\boldsymbol{k}, \omega) /(2 i) \operatorname{Im} G_{0}^{R}(\boldsymbol{k}, \omega) \\
& =\frac{2 \pi N_{\mathrm{F}} S_{0}\left(\gamma h_{\mathrm{ac}} / 2\right)^{2}}{\alpha \omega} \delta_{\boldsymbol{k}, \boldsymbol{0}} \delta(\omega-\Omega) .
\end{aligned}
$$

In the setup of the SSE, FI and SC are in equilibrium with temperatures $T_{\mathrm{FI}}$ and $T_{\mathrm{SC}}$, respectively. Using their Lehmann representation, we can prove the relations $[46,47]$

$$
\begin{aligned}
& \chi^{<}(\boldsymbol{q}, \omega)=2 i \operatorname{Im} \chi^{R}(\boldsymbol{q}, \omega) n_{\mathrm{B}}\left(\omega, T_{\mathrm{SC}}\right), \\
& G^{<}(\boldsymbol{k}, \omega)=2 i \operatorname{Im} G^{R}(\boldsymbol{k}, \omega) n_{\mathrm{B}}\left(\omega, T_{\mathrm{FI}}\right),
\end{aligned}
$$

where $n_{\mathrm{B}}(\omega, T)$ is the Bose distribution function defined as

$$
n_{\mathrm{B}}(\omega, T)=\frac{1}{e^{\hbar \omega / k_{\mathrm{B}} T}-1} .
$$

This result leads to the distribution functions of the FI and the SC [defined in Eqs. (20) and (21)] as

$$
\begin{gathered}
f^{\mathrm{SC}}(\boldsymbol{q}, \omega)=n_{\mathrm{B}}\left(\omega, T_{\mathrm{SC}}\right), \\
f^{\mathrm{FI}}(\boldsymbol{k}, \omega)=n_{\mathrm{B}}\left(\omega, T_{\mathrm{FI}}\right) .
\end{gathered}
$$

\section{SPIN CURRENT}

\section{A. Formulation}

The spin current at the SC-FI interface is defined by $\left\langle\hat{I}_{S}\right\rangle$, where $\langle\cdots\rangle$ denotes the statistical average and $\hat{I}_{S}$ is the operator for the spin current flowing from the SC to the FI, defined as

$$
\begin{aligned}
\hat{I}_{\mathrm{S}} & :=-\hbar \partial_{t}\left(s_{\mathrm{tot}}^{z}\right)=i\left[s_{\mathrm{tot}}^{z}, H\right], \\
s_{\mathrm{tot}}^{z} & :=\frac{1}{2} \sum_{\boldsymbol{k}}\left(c_{\boldsymbol{k} \uparrow}^{\dagger} c_{\boldsymbol{k} \uparrow}-c_{\boldsymbol{k} \downarrow}^{\dagger} c_{\boldsymbol{k} \downarrow}\right) .
\end{aligned}
$$

By substituting the expression for the system Hamiltonian, we obtain

$$
\hat{I}_{S}=-i \sum_{k, q}\left(\mathcal{T}_{k, q} S_{k}^{+} s_{q}^{-}-\text {H.c. }\right) .
$$

We consider the second-order perturbation calculation by taking $H_{\mathrm{FI}}+H_{\mathrm{SC}}$ as an unperturbed Hamiltonian and $H_{\mathrm{ex}}$ as a perturbation. The average of the spin-current operator is written as

$$
\begin{aligned}
\left\langle\hat{I}_{S}\right\rangle & =\operatorname{Re}\left[-2 i \sum_{\boldsymbol{k}, \boldsymbol{q}} \mathcal{T}_{\boldsymbol{k}, \boldsymbol{q}}\left\langle s_{\boldsymbol{q}}^{-} S_{\boldsymbol{k}}^{+}\right\rangle\right] \\
& =\lim _{t_{1}, t_{2} \rightarrow 0} \operatorname{Re}\left[-2 i \sum_{\boldsymbol{k}, \boldsymbol{q}} \mathcal{T}_{\boldsymbol{k}, \boldsymbol{q}}\left\langle s_{\boldsymbol{q}}^{-}\left(t_{2}\right) S_{\boldsymbol{k}}^{+}\left(t_{1}\right)\right\rangle\right],
\end{aligned}
$$

where the average $\langle\cdots\rangle$ is taken for the full Hamiltonian. By using the formal expression of perturbation expansion, the 


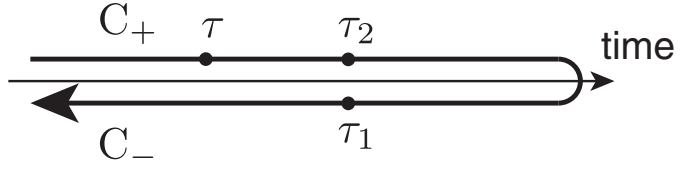

FIG. 2. The Keldysh contour $C$.

spin current can be rewritten as $[47,48]$

$$
\begin{aligned}
\left\langle\hat{I}_{S}\right\rangle= & \operatorname{Re}\left[-2 i \sum_{\boldsymbol{k}, \boldsymbol{q}} \mathcal{T}_{\boldsymbol{k}, \boldsymbol{q}}\left\langle T_{\mathrm{K}} s_{\boldsymbol{q}}^{-}\left(\tau_{2}\right) S_{\boldsymbol{k}}^{+}\left(\tau_{1}\right)\right.\right. \\
& \left.\left.\times \exp \left(-\frac{i}{\hbar} \int_{\mathrm{C}} d \tau H_{\mathrm{ex}}(\tau)\right)\right\rangle_{0}\right],
\end{aligned}
$$

where the average $\langle\cdots\rangle_{0}$ is now taken for the unperturbed Hamiltonian and $T_{\mathrm{K}}$ is the time-ordering operator on the time variable $\tau$ on the Keldysh contour $C$, which is composed of the forward path $C_{+}$running from $-\infty$ to $\infty$ and the backward path $C_{-}$from $\infty$ to $-\infty$ (see Fig. 2). We have put the time variables $\tau_{1}$ and $\tau_{2}$ on the contour $C_{-}$and $C_{+}$and have removed the limit operation for operator ordering.

Expanding the exponential operator in Eq. (37) and keeping the lowest-order term with respect to $H_{\mathrm{ex}}$, we obtain

$$
\begin{aligned}
\left\langle\hat{I}_{S}\right\rangle= & -\frac{2}{\hbar} \int_{\mathrm{C}} d \tau \operatorname{Re}\left[\sum_{\boldsymbol{k}, \boldsymbol{q}}\left|\mathcal{T}_{\boldsymbol{k}, \boldsymbol{q}}\right|^{2}\left\langle T_{\mathrm{K}} s_{\boldsymbol{q}}^{+}(\tau) s_{\boldsymbol{q}}^{-}\left(\tau_{2}\right)\right\rangle_{0}\right. \\
& \left.\times\left\langle T_{\mathrm{K}} S_{\boldsymbol{k}}^{+}\left(\tau_{1}\right) S_{\boldsymbol{k}}^{-}(\tau)\right\rangle_{0}\right] .
\end{aligned}
$$

Using the real-time representation [46-48], we can rewrite the spin current in terms of the dynamic spin susceptibilities of FI and $\mathrm{SC}$ as

$$
\begin{aligned}
\left\langle\hat{I}_{S}\right\rangle= & -2 \hbar \operatorname{Re} \int_{-\infty}^{\infty} d t \sum_{\boldsymbol{k}, \boldsymbol{q}}\left|\mathcal{T}_{\boldsymbol{k}, \boldsymbol{q}}\right|^{2} N_{\mathrm{S}} \\
& \times\left[\chi^{R}(\boldsymbol{q}, t) G^{<}(\boldsymbol{k},-t)+\chi^{<}(\boldsymbol{q}, t) G^{A}(\boldsymbol{k},-t)\right],
\end{aligned}
$$

where $G^{A}(\boldsymbol{k}, t)$ is the advanced component. Using the definitions of the distribution functions and performing the Fourier transformation for the dynamic spin susceptibilities, we obtain

$$
\begin{aligned}
\left\langle\hat{I}_{S}\right\rangle= & 4 \hbar \int \frac{d \omega}{2 \pi} \sum_{\boldsymbol{k}, \boldsymbol{q}}\left|\mathcal{T}_{\boldsymbol{k}, \boldsymbol{q}}\right|^{2} N_{\mathrm{S}} \operatorname{Im} \chi^{R}(\boldsymbol{q}, \omega) \\
& \times\left[-\operatorname{Im} G^{R}(\boldsymbol{k}, \omega)\right]\left[f^{\mathrm{FI}}(\boldsymbol{k}, \omega)-f^{\mathrm{SC}}(\boldsymbol{q}, \omega)\right] .
\end{aligned}
$$

Setting $\mathcal{T}_{k, q}=\mathcal{T}$ for simplicity, we obtain

$$
\begin{aligned}
\left\langle\hat{I}_{S}\right\rangle= & \hbar A \int \frac{d(\hbar \omega)}{2 \pi} \frac{1}{N_{\mathrm{S}} N_{\mathrm{F}}} \sum_{\boldsymbol{k}, \boldsymbol{q}} \operatorname{Im} \chi^{R}(\boldsymbol{q}, \omega) \\
& \times\left[-\operatorname{Im} G^{R}(\boldsymbol{k}, \omega)\right]\left[f^{\mathrm{FI}}(\boldsymbol{k}, \omega)-f^{\mathrm{SC}}(\boldsymbol{q}, \omega)\right],
\end{aligned}
$$

where $A=4|\mathcal{T}|^{2} N_{\mathrm{S}}^{2} N_{\mathrm{F}} / \hbar$.

\section{B. Spin pumping}

We first consider the case of spin pumping driven by microwave irradiation, keeping the same temperature for both
SC and FI. From Eq. (27), the difference of the distribution functions is given by

$f^{\mathrm{FI}}(\boldsymbol{k}, \omega)-f^{\mathrm{SC}}(\boldsymbol{q}, \omega)=\frac{2 \pi N_{F} S_{0}\left(\gamma h_{\mathrm{ac}} / 2\right)^{2}}{\alpha \omega} \delta_{\boldsymbol{k}, \mathbf{0}} \delta(\omega-\Omega)$.

The spin current generated by SP is then given by

$$
\begin{gathered}
I_{S}^{\mathrm{SP}}=\hbar A g(\Omega) \operatorname{Im} \chi_{\mathrm{loc}}^{R}(\Omega), \\
g(\Omega):=\frac{\left(\gamma h_{\mathrm{ac}} S_{0}\right)^{2} / 2}{\left(\Omega-\omega_{0}\right)^{2}+\alpha^{2} \Omega^{2}},
\end{gathered}
$$

where the local spin susceptibility $\chi_{\mathrm{loc}}^{R}(\omega)$ is given by Eqs. (13) and (14) and $\omega_{0}=\gamma h_{\mathrm{dc}}$ is the angular frequency of the spin precession.

For the normal-metal case $(\Delta=0)$, we obtain for the spin current, using Eq. (15),

$$
I_{\mathrm{S}}^{\mathrm{SP}, \mathrm{N}}=\pi \hbar A g(\Omega) N\left(\epsilon_{\mathrm{F}}\right)^{2} \hbar \Omega,
$$

which is temperature independent for arbitrary values of $\Omega$. We will use this expression as a normalization factor to compare the results at finite $\Delta$ for various frequencies $\Omega$.

Before showing the results obtained in the superconducting case, we point out that in the low-frequency limit $(\Omega \rightarrow$ 0 ), the expression for the spin current generated by SP is similar to the one obtained when computing the nuclear spin resonance (NMR) signal [45]. It is known in the theory of the NMR measurement that the BCS singularity in the density of states leads to a coherence peak below the SC transition temperature [49,50]. As a consequence, one can expect a similar coherence peak in the temperature dependence of the spin current at low frequency. However, the spin current contains more information than the NMR expression since $\Omega$ can be controlled arbitrarily up to high frequencies of the order of the transition temperature $T_{\mathrm{c}}$.

In Fig. 3, we show the temperature dependence of the spin current induced by spin pumping. Here, the temperatures of both FI and SC are set to $T$, and the spin current is normalized by the value obtained for the normal-metal case $I_{\mathrm{S}}^{\mathrm{SP}, \mathrm{N}}$. For low excitation frequency $\Omega$, the temperature dependence of $I_{\mathrm{S}}^{\mathrm{SP}}$ clearly shows a coherence peak below the $\mathrm{SC}$ transition temperature $T_{\mathrm{c}}$, as expected. For $\hbar \Omega<2 \Delta(T=$ $0) \simeq 3.54 k_{\mathrm{B}} T_{\mathrm{c}}$, the spin current is strongly reduced at low temperatures $\left[k_{\mathrm{B}} T \ll 2 \Delta(T)\right]$ because spin-flip excitations in the SC are suppressed due to the energy gap $2 \Delta$ in the one-electron excitation spectrum. As $\Omega$ increases, the coherence peak becomes insignificant, while a kink appears at the temperature satisfying $2 \Delta(T)=\hbar \Omega$. For $\hbar \Omega>2 \Delta(T=0)$, the spin current shows a plateau at low temperature corresponding to its zero-temperature value, ultimately recovering the normal-state value (dashed line) as $\hbar \Omega$ is increased further.

\section{Spin Seebeck effect}

We now turn to the alternative technique for generating a spin current, namely, the spin Seebeck effect, which relies on the presence of a temperature gradient between the FI and SC layers. Using Eqs. (31) and (32), the spin current induced by 

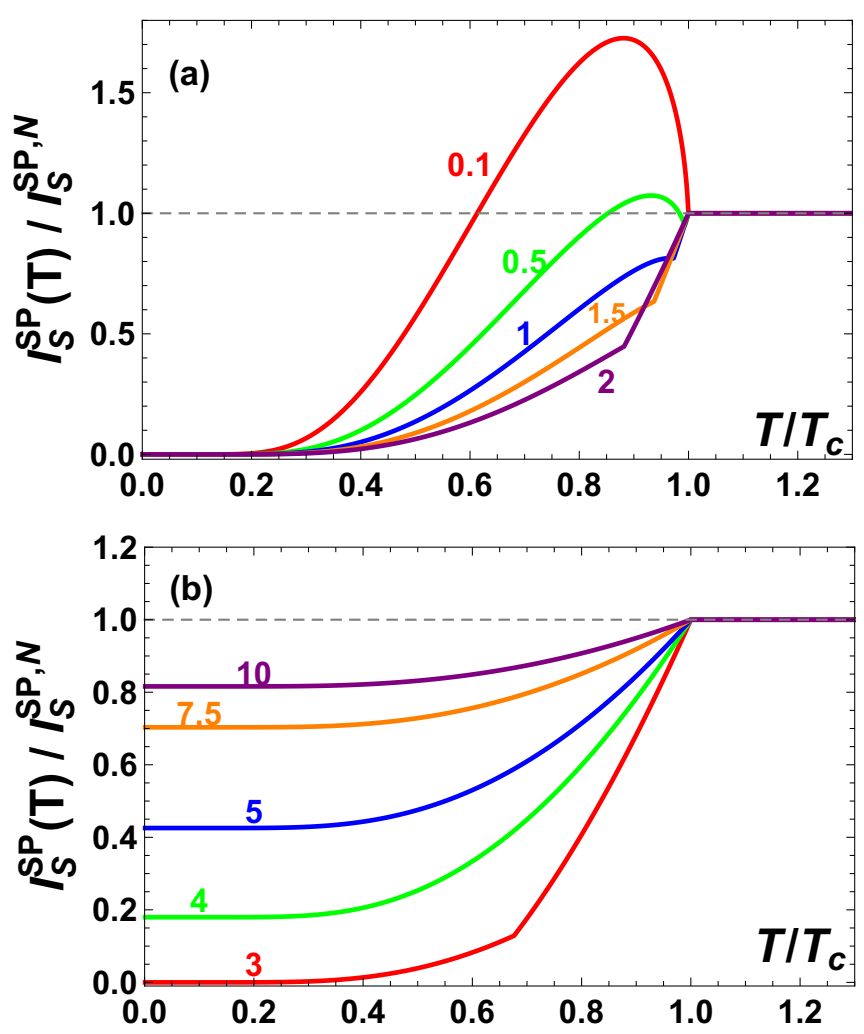

FIG. 3. Temperature dependence of the spin current induced by spin pumping $I_{S}^{S P}(T)$, normalized by the current obtained in the normal case $I_{S}^{S P, N}(T)$, for different values of $\hbar \Omega / T_{\mathrm{c}}$, as indicated near each curve. (a) shows $\hbar \Omega / T_{\mathrm{c}}$ from 0.1 to 2 . (b) shows $\hbar \Omega / T_{\mathrm{c}}$ from 3.0 to 10 .

the spin Seebeck effect is given by

$$
\begin{aligned}
I_{\mathrm{S}}^{\mathrm{SSE}}= & \hbar A \int \frac{d(\hbar \omega)}{2 \pi} \operatorname{Im} \chi_{\mathrm{loc}}^{R}(\omega)\left[-\operatorname{Im} G_{\mathrm{loc}}^{R}(\omega)\right] \\
& \times\left[n_{\mathrm{B}}\left(\omega, T_{\mathrm{FI}}\right)-n_{\mathrm{B}}\left(\omega, T_{\mathrm{SC}}\right)\right],
\end{aligned}
$$

where $G_{\mathrm{loc}}^{R}(\omega):=N_{\mathrm{F}}^{-1} \sum_{k} G^{R}(\boldsymbol{k}, \omega)$ is the local spin susceptibility in the FI. For simplicity, we consider the spin Seebeck effect up to the linear term with respect to the temperature difference $\delta T=T_{\mathrm{FI}}-T_{\mathrm{SC}}$ :

$$
\begin{aligned}
\frac{I_{\mathrm{S}}^{\mathrm{SSE}}}{I_{\mathrm{S}, 0}^{\mathrm{SSE}}} & =\int_{E_{0}}^{E_{\mathrm{M}}} d E D_{\mathrm{M}}(E) F(E) \frac{\left(E / 2 k_{\mathrm{B}} T\right)^{2}}{\sinh ^{2}\left(E / 2 k_{\mathrm{B}} T\right)}, \\
F(E):= & \operatorname{Im} \chi_{\mathrm{loc}}^{R}(E / \hbar) / \operatorname{Im} \chi_{\mathrm{loc}, \Delta=0}^{R}(E / \hbar) \\
= & \int_{-\infty}^{\infty} d E^{\prime}\left[1+\frac{\Delta^{2}}{E^{\prime}\left(E^{\prime}+E\right)}\right] \\
& \times\left[\frac{f\left(E^{\prime}\right)-f\left(E^{\prime}+E\right)}{E}\right] D\left(E^{\prime}\right) D\left(E^{\prime}+E\right),
\end{aligned}
$$

where $T=T_{\mathrm{SC}} \simeq T_{\mathrm{FI}}$ and $I_{\mathrm{S}, 0}^{\mathrm{SSE}}=\hbar A S_{0} k_{\mathrm{B}} \delta T N\left(\epsilon_{\mathrm{F}}\right)^{2}$. The density of states per site for a magnon is given by

$$
\begin{aligned}
D_{\mathrm{M}}(E) & :=\frac{1}{N_{\mathrm{F}}} \sum_{k} \delta\left(E-\hbar \omega_{\boldsymbol{k}}\right) \\
& =-\left(2 \pi S_{0}\right)^{-1} \operatorname{Im} G_{\mathrm{loc}}^{R}(E / \hbar),
\end{aligned}
$$

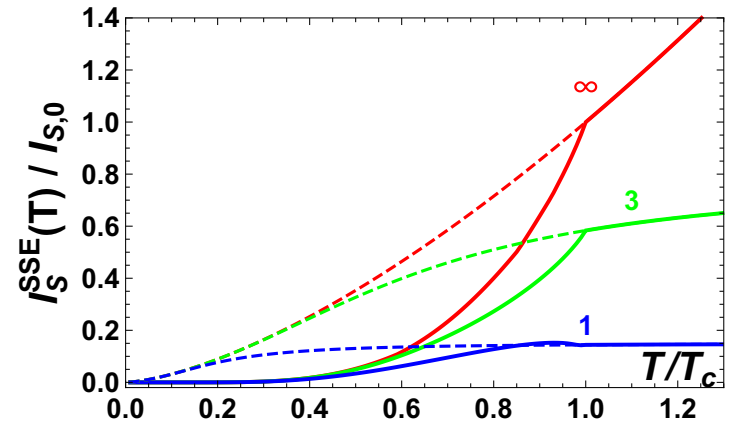

FIG. 4. Temperature dependence of the spin current induced by the spin Seebeck effect $I_{\mathrm{S}}^{\mathrm{SSE}}$, normalized by $I_{\mathrm{S}, 0}=$ $I_{\mathrm{S}, 0}^{\mathrm{SSE}} \eta\left(k_{\mathrm{B}} T_{\mathrm{c}} / E_{\mathrm{M}}\right)^{3 / 2}$, for SCs (solid lines) and normal metals (dashed lines) with $E_{\mathrm{M}} / k_{\mathrm{B}} T_{\mathrm{c}}=\infty, 3$, and 1 (as indicated near each curve), where $E_{\mathrm{M}}$ is the high-energy cutoff of the magnon density of states. As we consider $E_{0} \ll k_{\mathrm{B}} T_{\mathrm{c}}$, the Zeeman energy $E_{0}$ is set to zero for simplicity.

taking the limit $\alpha \rightarrow 0$, and $E_{\mathrm{M}}\left(\gg E_{0}\right)$ is the high-energy cutoff of the magnon dispersion relation, which is of the order of the exchange interaction in the FI. Under a uniform magnetic field, the local spin susceptibility is evaluated for the parabolic magnon dispersion as $D_{\mathrm{M}}(E)=(3 / 2)\left(E-E_{0}\right)^{1 / 2} E_{\mathrm{M}}^{-3 / 2}$. For normal metals $(\Delta=0)$, the spin current at low temperatures $\left(k_{\mathrm{B}} T \ll E_{\mathrm{M}}\right)$ is given by $I_{\mathrm{S}}^{\mathrm{SSE}} / I_{\mathrm{S}, 0}^{\mathrm{SSE}}=\eta\left(k_{\mathrm{B}} T / E_{\mathrm{M}}\right)^{3 / 2}$, where $\eta \simeq 6.69$ is a numerical factor.

In Fig. 4, we show the temperature dependence of $I_{\mathrm{S}}^{\mathrm{SSE}}$. The solid and dashed lines show $I_{\mathrm{S}}^{\mathrm{SSE}}$ for the SC and the normal metal $(\Delta=0)$, respectively. For simplicity, the Zeeman energy is set to zero by assuming that it is much smaller than $k_{\mathrm{B}} T$. When $E_{\mathrm{M}}$ is much larger than $k_{\mathrm{B}} T_{\mathrm{c}}$, the spin current monotonically decreases as the temperature is lowered. Below the transition temperature $T_{\mathrm{c}}$, the spin current at the FI-SC interface is suppressed due to the opening of the energy gap in the SC. When $E_{\mathrm{M}}$ is comparable to $k_{\mathrm{B}} T_{\mathrm{c}}$, the spin current shows a small maximum below $T_{\mathrm{c}}$ and saturates above $T_{\mathrm{c}}$.

\section{SPIN-CURRENT NOISE}

The noise of the pure spin current has been studied for an interface between a FI and a nonmagnetic metal [43,51,52], as well as for several hybrid nanostructures [53-56]. It includes useful information on spin transport, as suggested from studies of the (electronic) current noise [57]. In this section, we calculate the spin-current noise for the FI-SC interface.

\section{A. Formulation}

The noise power of the pure spin current is defined as [43]

$$
\mathcal{S}:=\lim _{\mathcal{T} \rightarrow \infty} \frac{1}{\mathcal{T}} \int_{0}^{\mathcal{T}} d t_{1} \int_{0}^{\mathcal{T}} d t_{2} \frac{1}{2}\left\langle\left\{\hat{I}_{S}\left(t_{1}\right), \hat{I}_{S}\left(t_{2}\right)\right\}\right\rangle,
$$

where $\hat{I}_{S}(t):=e^{i H t} I_{S} e^{-i H t}$ and $\{A, B\}=A B+B A$. The spincurrent noise is calculated within the second-order perturbation calculation with respect to $H_{\mathrm{ex}}$ as

$$
\begin{aligned}
\mathcal{S}= & \hbar^{2} \int_{-\infty}^{\infty} \frac{d \omega}{2 \pi} \sum_{\boldsymbol{k}, \boldsymbol{q}}\left|\mathcal{T}_{\boldsymbol{k}, \boldsymbol{q}}\right|^{2} N_{\mathrm{S}}\left[\chi^{<}(\boldsymbol{q}, \omega) G^{>}(\boldsymbol{k}, \omega)\right. \\
& \left.+\chi^{>}(\boldsymbol{q}, \omega) G^{<}(\boldsymbol{k}, \omega)\right] .
\end{aligned}
$$


Using $\mathcal{T}_{\boldsymbol{k}, \boldsymbol{q}}=\mathcal{T}$, Eqs. (20) and (21), and the relations

$$
\begin{aligned}
& \chi^{>}(\boldsymbol{q}, \omega) /(2 i) \operatorname{Im} \chi^{R}(\boldsymbol{q}, \omega)=1+f^{\mathrm{SC}}(\boldsymbol{q}, \omega), \\
& G^{>}(\boldsymbol{k}, \omega) /(2 i) \operatorname{Im} G^{R}(\boldsymbol{k}, \omega)=1+f^{\mathrm{FI}}(\boldsymbol{k}, \omega),
\end{aligned}
$$

the spin-current noise is calculated as

$$
\begin{aligned}
\mathcal{S}= & \hbar^{2} A \int \frac{d(\hbar \omega)}{2 \pi} \frac{1}{N_{\mathrm{F}} N_{\mathrm{S}}} \sum_{\boldsymbol{k}, \boldsymbol{q}}\left[-\operatorname{Im} G^{R}(\boldsymbol{k}, \omega)\right] \operatorname{Im} \chi^{R}(\boldsymbol{q}, \omega) \\
& \times\left[f^{\mathrm{SC}}(\boldsymbol{q}, \omega)\left[1+f^{\mathrm{FI}}(\boldsymbol{k}, \omega)\right]\right. \\
& \left.+\left[1+f^{\mathrm{SC}}(\boldsymbol{q}, \omega)\right] f^{\mathrm{FI}}(\boldsymbol{k}, \omega)\right] .
\end{aligned}
$$

In the absence of both the external microwave excitation and the temperature gradient, the noise power is determined by the equilibrium noise:

$$
\mathcal{S}^{\mathrm{eq}}=2 \hbar^{2} A \int_{-\infty}^{\infty} \frac{d(\hbar \omega)}{2 \pi} \frac{\operatorname{Im} \chi_{\mathrm{loc}}^{R}(\omega)\left[-\operatorname{Im} G_{\mathrm{loc}}^{R}(\omega)\right]}{4 \sinh ^{2}\left(\hbar \omega / 2 k_{\mathrm{B}} T\right)} .
$$

Under the microwave radiation, the noise power is calculated from Eq. (42) as

$$
\begin{gathered}
\mathcal{S}=\mathcal{S}^{\mathrm{eq}}+\mathcal{S}^{\mathrm{SP}}, \\
\mathcal{S}^{\mathrm{SP}}=\hbar \operatorname{coth}\left(\frac{\hbar \Omega}{2 k_{\mathrm{B}} T}\right) I_{\mathrm{S}}^{\mathrm{SP}},
\end{gathered}
$$

where $\mathcal{S}^{\mathrm{SP}}$ is the nonequilibrium noise induced by spin pumping. While the nonequilibrium noise can similarly be induced by SSE, we do not discuss it here as it requires us to consider a large temperature gradient.

\section{B. Estimate}

As in the metal-FI bilayer system [43,51,52], the noise power of the pure spin current includes useful information also in the case of the SC-FI interface. At low temperatures $\left(k_{\mathrm{B}} T \ll \hbar \Omega\right)$, the ratio $\mathcal{S}^{\mathrm{SP}} / I_{\mathrm{S}}^{\mathrm{SP}}$ approaches $\hbar$, indicating that each magnon excitation carries the angular momentum $\hbar$. At high temperatures $\left(k_{\mathrm{B}} T \gg \hbar \Omega\right)$, this ratio becomes proportional to $k_{\mathrm{B}} T$ due to the nature of the Bose statistics. To illustrate their temperature dependence, we estimate and compare the noise powers, $\mathcal{S}^{\mathrm{eq}}$ and $\mathcal{S}^{\mathrm{SP}}$, in realistic experiments. We use the parameters of the spin-pumping experiment for YIG [29]: $\alpha=6.7 \times 10^{-5}, S_{0}=16, h_{\mathrm{ac}}=0.11 \mathrm{Oe}, \gamma=1.76 \times$ $10^{7} \mathrm{Oe}^{-1} \mathrm{~s}^{-1}$, and $\Omega / 2 \pi=9.4 \mathrm{GHz}$. We consider $\mathrm{NbN}$ for the $\mathrm{SC}\left(T_{\mathrm{c}} \simeq 10 \mathrm{~K}\right)$ and set $D=532 \mathrm{meV} \AA^{2}$ following Ref. [58]. Figure 5 shows the results for the noise power, normalized by $\mathcal{S}_{0}=\mathcal{S}^{\mathrm{SP}}(T=0)$ for normal metals. For this parameter set, the nonequilibrium noise associated with spin pumping is much larger than the equilibrium noise. For both $\mathcal{S}^{\text {eq }}$ and $\mathcal{S}^{\text {sP }}$, the temperature dependence peaks below the superconducting transition temperature.

\section{EXPERIMENTAL SETUP FOR DETECTION}

In the previous sections, we evaluated the spin current and its noise at the FI-SC interface. For their experimental detection, we need to consider a setup for converting the spin imbalance induced by the spin current into a charge signal.

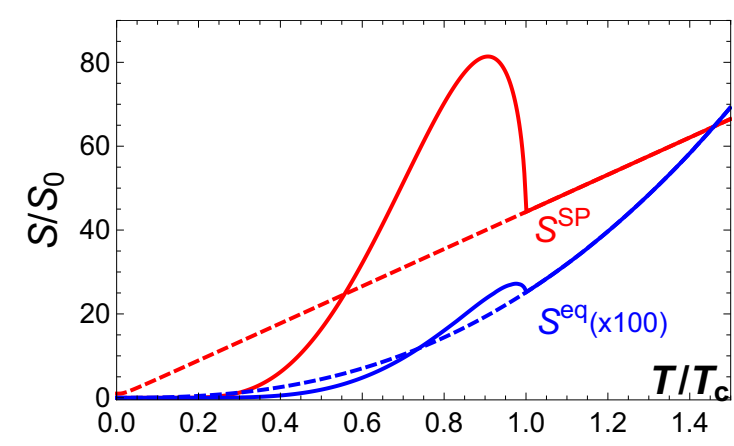

FIG. 5. Temperature dependence of the equilibrium noise $\mathcal{S}^{\text {eq }}$ and the nonequilibrium noise in the spin-pumping case $\mathcal{S}^{\mathrm{SP}}$ for SCs (solid lines) and normal metals (dashed lines). The noise power is normalized by the nonequilibrium noise in the spin-pumping case $\mathcal{S}_{0}$ for the normal metals at $T=0$. For better visualization, data for the equilibrium noise have been multiplied by 100 .

There are several ways to perform such a spin-charge conversion. Here, we explain one possible way using the ISHE. It was theoretically predicted that such spin current flowing in a SC can be detected by the ISHE [35,36]. Indeed, a giant signal of the ISHE was recently observed by spin injection from ferromagnetic metals into an $s$-wave superconductor $\mathrm{NbN}$ using the technique of the lateral spin valve [37].

Let us consider spin injection into a SC wire with a width $w$ and a length $2 d$ from a FI at $x=0$ (see Fig. 6). By spin-orbit interaction in the SC, the spin current $I_{S}$ is converted into a quasiparticle current $I_{Q}$ in the direction perpendicular to both $I_{S}$ and the ordered spin in the FI $S$. This quasiparticle current induces a charge imbalance in the SC and produces a voltage between the two edges located at $x= \pm d$. The amplitude of the ISHE voltage depends on the spin relaxation in the SC as well as the spin Hall angle, so that the coefficient between the spin current at the interface and the ISHE voltage is, in general, temperature dependent. Here, we introduce a simple formula employed in Ref. [37]:

$$
\begin{gathered}
V_{\mathrm{ISHE}}=\frac{|e|}{\hbar} I_{S} \frac{x}{w}\left[a \frac{\rho_{x x}}{2 f_{0}(\Delta)}+b\left(\frac{\rho_{x x}}{2 f_{0}(\Delta)}\right)^{2}\right] e^{-d / \lambda_{Q},} \\
f_{0}(\Delta)=\frac{1}{e^{\Delta / k_{\mathrm{B}} T}+1} .
\end{gathered}
$$

This expression for the ISHE voltage has been derived assuming an extrinsic spin Hall effect due to spin-orbit scattering in the SC. Here, $\lambda_{Q}$ is the charge relaxation length, $a$ and $b$ are coefficients determined by the strength of the skew scattering

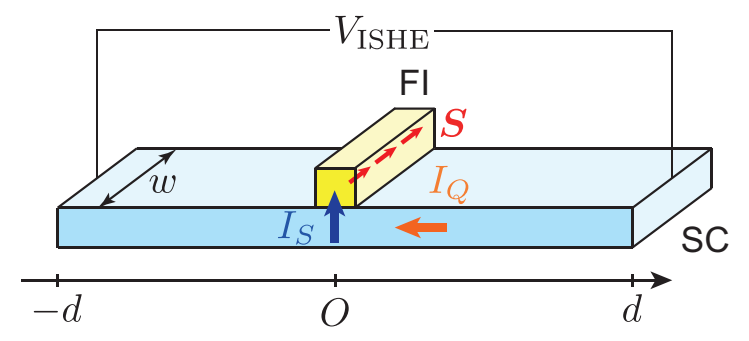

FIG. 6. A setup for detection of the spin current using the inverse spin Hall effect. 
and side jump, respectively, and $\rho_{x x}$ is the resistivity of the $\mathrm{SC}$. The correction due to nonuniform current distribution is represented by a shunting length $x$, which is determined by $w$, $\lambda_{Q}$, and the shape of the junction [37]. Combining Eqs. (58) and (59) with careful determination of the parameters, we can obtain $I_{S}$ from the measurement of $V_{\text {ISHE }}$. In principle, the spin-current noise can also be measured within the same kind of setup via the fluctuations of $V_{\text {ISHE }}$ [43].

\section{SUMMARY}

In summary, we discussed the spin current and the spincurrent noise for a bilayer system composed of a superconductor and a ferromagnetic insulator. The spin current induced by spin pumping has a maximum below the transition temperature when the pumping frequency $\Omega$ is much smaller than $k_{\mathrm{B}} T_{c} / \hbar$. As the ratio $\hbar \Omega / k_{\mathrm{B}} T_{c}$ increases, the peak disappears, and the spin current at low temperatures is enhanced. We also discussed the spin current induced by the spin Seebeck effect and the noise power of the pure spin current. Our study provides a fundamental basis for the application of spintronics using superconductors. Extension to spin injection from antiferromagnetic insulators is left for a future problem [59-62].

\section{ACKNOWLEDGMENTS}

The authors are grateful to S. Takei, Y. Niimi, Y.-C. Otani, K. Kobayashi, and T. Arakawa for useful discussions and comments. This work is financially supported by KAKENHI (Grants No. 26103006, No. JP26220711, No. JP16H04023, and No. JP17H02927) from MEXT and JSPS, Japan. This work has been supported by the Excellence Initiative of AixMarseille University (AMIDEX), a French "investissements d'avenir" program.

\section{APPENDIX A: EFFECT OF IMPURITY SCATTERING}

Here, we explain that the diffusive behavior of conduction electrons, which is taken into account in Ref. [38], can be neglected in the calculation of $\operatorname{Im} \chi_{\text {loc }}^{R}(\omega)$ following Ref. [42]. We neglect the Coulomb interaction effect discussed in Ref. [42] for simplicity. For a qualitative discussion, it is convenient to start with the interpolation formula (Eq. (6) in Ref. [42])

$$
\chi_{D}^{R}(\boldsymbol{q}, \omega) \simeq \chi_{0}(\boldsymbol{q}, \omega) \frac{\mathcal{D} q^{2}}{\mathcal{D} q^{2}-i \omega},
$$

where $q=|\boldsymbol{q}|, \chi_{0}(\boldsymbol{q}, \omega)$ is the spin susceptibility per volume of the electron gas, $\mathcal{D}=v_{F} l / 3$ is the diffusion constant, $l=$ $v_{F} \tau$ is the mean free path, $v_{\mathrm{F}}$ is the Fermi velocity, and $\tau$ is the relaxation time. The leading behavior for small $\omega$ is (see
Eq. (7) in Ref. [42])

$$
\frac{\operatorname{Im} \chi(\boldsymbol{q}, \omega)}{\hbar \omega}=\frac{N\left(\epsilon_{\mathrm{F}}\right)}{\hbar}\left(\frac{\pi}{2 v_{F} q}+\frac{1}{\mathcal{D} q^{2}}\right) \quad\left(0<q<2 k_{F}\right)
$$

where $k_{\mathrm{F}}$ is the Fermi wave number. Then, the local spin susceptibility is calculated as

$$
\begin{aligned}
\frac{\operatorname{Im} \chi_{\mathrm{loc}}(\omega)}{\hbar \omega} & =\int \frac{d^{3} \boldsymbol{q}}{(2 \pi)^{3}} \frac{\operatorname{Im} \chi(\boldsymbol{q}, \omega)}{\hbar \omega} \\
& =2 \pi N\left(\epsilon_{F}\right)^{2}\left(\frac{1}{2}+\frac{3}{\pi k_{F} l}\right) .
\end{aligned}
$$

Since $k_{F} l \gg 1$ for usual metals, the second term due to the diffusive Green's function is usually a correction. Therefore, the leading contribution is obtained only by considering a clean system without impurities. For superconductors, a similar discussion leads to the same conclusion.

\section{APPENDIX B: SPIN SUSCEPTIBILITY OF THE SC}

The dynamic spin susceptibility of the SC is calculated in the standard BCS theory as [45]

$$
\begin{aligned}
\chi^{R}(\boldsymbol{q}, \omega)= & \frac{1}{N_{\mathrm{S}}} \sum_{\boldsymbol{k}} \sum_{\lambda= \pm 1} \sum_{\lambda^{\prime}= \pm 1}\left[\frac{1}{4}+\frac{\xi \xi^{\prime}+\Delta^{2}}{4 E_{\lambda} E_{\lambda^{\prime}}^{\prime}}\right] \\
& \times \frac{f\left(E_{\lambda^{\prime}}^{\prime}\right)-f\left(E_{\lambda}\right)}{\hbar \omega+i \delta+E_{\lambda}-E_{\lambda^{\prime}}^{\prime}},
\end{aligned}
$$

where $\quad \xi=\xi_{k}, \quad \xi^{\prime}=\xi_{k+q}, \quad E_{\lambda}=\lambda \sqrt{\Delta^{2}+\xi^{2}}, \quad E_{\lambda^{\prime}}^{\prime}=$ $\lambda^{\prime} \sqrt{\Delta^{2}+\xi^{\prime 2}}$, and $f(E)=\left[\exp \left(E / k_{\mathrm{B}} T\right)+1\right]^{-1}$ is the Fermi distribution function. For the normal state $(\Delta=0)$, the spin susceptibility becomes

$$
\chi^{R}(\boldsymbol{q}, \omega)=\frac{1}{N_{\mathrm{S}}} \sum_{\boldsymbol{k}} \frac{f\left(\xi_{\boldsymbol{k}+\boldsymbol{q}}\right)-f\left(\xi_{\boldsymbol{k}}\right)}{\hbar \omega+i \delta+\xi_{\boldsymbol{k}}-\xi_{\boldsymbol{k}+\boldsymbol{q}}} .
$$

The imaginary part of the local spin susceptibility is obtained for the $\mathrm{SC}$ as

$$
\begin{aligned}
\operatorname{Im} \chi_{\mathrm{loc}}^{R}(\omega)= & -\frac{\pi}{N_{\mathrm{S}}^{2}} \sum_{k, \boldsymbol{k}^{\prime}} \sum_{\lambda, \lambda^{\prime}}\left[\frac{1}{4}+\frac{\xi \xi^{\prime}}{4 E_{\lambda} E_{\lambda^{\prime}}^{\prime}}\right] \\
& \times\left[f\left(E_{\lambda^{\prime}}^{\prime}\right)-f\left(E_{\lambda}\right)\right] \delta\left(\hbar \omega+E_{\lambda}-E_{\lambda^{\prime}}^{\prime}\right),
\end{aligned}
$$

where $\xi^{\prime}=\xi_{\boldsymbol{k}^{\prime}}$ and $E_{\lambda^{\prime}}^{\prime}=\lambda^{\prime} E_{\boldsymbol{k}^{\prime}}$. For $\hbar \omega \ll \epsilon_{\mathrm{F}}\left(\epsilon_{\mathrm{F}}\right.$ is the Fermi energy), we can replace the wave number summation according to

$$
\frac{1}{N_{\mathrm{S}}} \sum_{\boldsymbol{k}}(\cdots) \rightarrow N\left(\epsilon_{\mathrm{F}}\right) \int_{-\infty}^{\infty} d \xi(\cdots),
$$

where $N\left(\epsilon_{\mathrm{F}}\right)$ is the density of states per spin and per unit cell. Changing the integral variable from $\xi$ to $E=\sqrt{\Delta^{2}+\xi^{2}}$, we finally obtain Eqs. (13) and (14).
[1] P. M. Tedrow, R. Meservey, and P. Fulde, Phys. Rev. Lett. 25, 1270 (1970).

[2] R. Meservey and P. M. Tedrow, Phys. Rep. 238, 173 (1994).

[3] I. Žutić, J. Fabian, and S. Das Sarma, Rev. Mod. Phys. 76, 323 (2004).
[4] J. Linder and J. W. A. Robinson, Nat. Phys. 11, 307 (2015).

[5] S. A. Kivelson and D. S. Rokhsar, Phys. Rev. B 41, 11693(R) (1990).

[6] H. L. Zhao and S. Hershfield, Phys. Rev. B 52, 3632 (1995). 
[7] F. S. Bergeret, M. Silaev, P. Virtanen, and T. T. Heikkilä, Rev. Mod. Phys. 90, 041001 (2018).

[8] S. Das Sarma, J. Fabiana, X. Hua, and I. Žutić, Solid State Commun. 119, 207 (2001).

[9] F. Hübler, M. J. Wolf, D. Beckmann, and H. v. Löhneysen, Phys. Rev. Lett. 109, 207001 (2012).

[10] C. H. L. Quay, D. Chevallier, C. Bena, and M. Aprili, Nat. Phys. 9, 84 (2013).

[11] M. J. Wolf, F. Hübler, S. Kolenda, H. v. Löhneysen, and D. Beckmann, Phys. Rev. B 87, 024517 (2013).

[12] T. Wakamura, N. Hasegawa, K. Ohnishi, Y. Niimi, and Y. C. Otani, Phys. Rev. Lett. 112, 036602 (2014).

[13] T. Yamashita, S. Takahashi, H. Imamura, and S. Maekawa, Phys. Rev. B 65, 172509 (2002).

[14] S. Takahashi and S. Maekawa, Phys. Rev. B 67, 052409 (2003).

[15] J. P. Morten, A. Brataas, and W. Belzig, Phys. Rev. B 70, 212508 (2004).

[16] J. P. Morten, A. Brataas, and W. Belzig, Phys. Rev. B 72, 014510 (2005).

[17] M. Silaev, P. Virtanen, F. S. Bergeret, and T. T. Heikkilä, Phys. Rev. Lett. 114, 167002 (2015).

[18] F. Aikebaier, M. A. Silaev, and T. T. Heikkilä, Phys. Rev. B 98, 024516 (2018).

[19] K. Uchida, S. Takahashi, K. Harii, J. Ieda, W. Koshibae, K. Ando, S. Maekawa, and E. Saitoh, Nature (London) 455, 778 (2008).

[20] C. M. Jaworski, J. Yang, S. Mack, D. D. Awschalom, J. P. Heremans, and R. C. Myers, Nat. Mater. 9, 898 (2010).

[21] K. Uchida, J. Xiao, H. Adachi, J. Ohe, S. Takahashi, J. Ieda, T. Ota, Y. Kajiwara, H. Umezawa, H. Kawai, G. E. W. Bauer, S. Maekawa, and E. Saitoh, Nat. Mater. 9, 894 (2010).

[22] J. Xiao, G. E. W. Bauer, K.-c. Uchida, E. Saitoh, and S. Maekawa, Phys. Rev. B 81, 214418 (2010).

[23] H. Adachi, J.-i. Ohe, S. Takahashi, and S. Maekawa, Phys. Rev. B 83, 094410 (2011).

[24] H. Adachi, K. Uchida, E. Saitoh, and S. Maekawa, Rep. Prog. Phys. 76, 036501 (2013).

[25] Y. Ohnuma, M. Matsuo, and S. Maekawa, Phys. Rev. B 96, 134412 (2017).

[26] Y. Tserkovnyak, A. Brataas, and G. E. W. Bauer, Phys. Rev. Lett. 88, 117601 (2002).

[27] J. König and J. Martinek, Phys. Rev. Lett. 90, 166602 (2003).

[28] E. Saitoh, M. Ueda, H. Miyajima, and G. Tatara, Appl. Phys. Lett. 88, 182509 (2006).

[29] Y. Kajiwara, K. Harii, S. Takahashi, J. Ohe, K. Uchida, M. Mizuguchi, H. Umezawa, H. Kawai, K. Ando, K. Takanashi, S. Maekawa, and E. Saitoh, Nature (London) 464, 262 (2010).

[30] Y. Ohnuma, H. Adachi, E. Saitoh, and S. Maekawa, Phys. Rev. B 89, 174417 (2014).

[31] K.-R. Jeon, C. Ciccarelli, A. J. Ferguson, H. Kurebayashi, L. F. Cohen, X. Montiel, M. Eschrig, J. W. A. Robinson, and M. G. Blamire, Nat. Mater. 17, 499 (2018).

[32] K.-R. Jeon, C. Ciccarelli, H. Kurebayashi, J. Wunderlich, L. F. Cohen, S. Komori, J. W. A. Robinson, and M. G. Blamire, Phys. Rev. Appl. 10, 014029 (2018).

[33] Y. Yao, Q. Song, Y. Takamura, J. P. Cascales, W. Yuan, Y. Ma, Y. Yun, X. C. Xie, J. S. Moodera, and W. Han, Phys. Rev. B 97, 224414 (2018).
[34] M. Umeda, Y. Shiomi, T. Kikkawa, T. Niizeki, J. Lustikova, S. Takahashi, and E. Saitoh, Appl. Phys. Lett. 112, 232601 (2018).

[35] S. Takahashi and S. Maekawa, Phys. Rev. Lett. 88, 116601 (2002).

[36] S. Takahashi and S. Maekawa, Sci. Technol. Adv. Mater. 9, 014105 (2008).

[37] T. Wakamura, H. Akaike, Y. Omori, Y. Niimi, S. Takahashi, A. Fujimaki, S. Maekawa, and Y. Otani, Nat. Mater. 14, 675 (2015).

[38] M. Inoue, M. Ichioka, and H. Adachi, Phys. Rev. B 96, 024414 (2017).

[39] A. A. Abrikosov and L. P. Gor'kov, Zh. Eksp. Teor. Fiz. 42, 1088 (1962) [Sov. Phys. JETP 15, 752 (1962)].

[40] L. P. Gor'kov and A. I. Rusinov, Zh. Eksp. Teor. Fiz. 46, 1361 (1964) [Sov. Phys. JETP 19, 922 (1964)].

[41] P. Fulde and A. Luther, Phys. Rev. 175, 337 (1968).

[42] B. S. Shastry and E. Abrahams, Phys. Rev. Lett. 72, 1933 (1994).

[43] M. Matsuo, Y. Ohnuma, T. Kato, and S. Maekawa, Phys. Rev. Lett. 120, 037201 (2018).

[44] T. Holstein and H. Primakoff, Phys. Rev. 58, 1098 (1940).

[45] P. Coleman, Introduction to Many-Body Physics (Cambridge University Press, Cambridge, 2015).

[46] H. Bruus and K. Flensberg, Many-Body Quantum Theory in Condensed Matter Physics: An Introduction (Oxford University Press, Oxford, 2004).

[47] G. Stefanucci and R. van Leeuwen, Nonequilibrium ManyBody Theory of Quantum Systems: A Modern Introduction (Cambridge University Press, Cambridge, 2013).

[48] J. Rammer and H. Smith, Rev. Mod. Phys. 58, 323 (1986).

[49] L. C. Hebel and C. P. Slichter, Phys. Rev. 113, 1504 (1959).

[50] Y. Masuda and A. G. Redfield, Phys. Rev. 125, 159 (1962).

[51] A. Kamra and W. Belzig, Phys. Rev. Lett. 116, 146601 (2016).

[52] A. Kamra and W. Belzig, Phys. Rev. B 94, 014419 (2016).

[53] J. Aftergood and S. Takei, Phys. Rev. B 97, 014427 (2018).

[54] D. G. Joshi, A. P. Schnyder, and S. Takei, Phys. Rev. B 98, 064401 (2018).

[55] K. Nakata, Y. Ohnuma, and M. Matsuo, Phys. Rev. B 98, 094430 (2018).

[56] J. Aftergood, M. Trif, and S. Takei, arXiv:1901.00933.

[57] Ya. M. Blanter and M. Büttiker, Phys. Rep. 336, 1 (2000); T. Martin, in Nanophysics: Coherence and Transport, edited by H. Bouchiat, Y. Gefen, S. Guéron, G. Montambaux, and J. Dalibard (Elsevier, Amsterdam, 2005).

[58] A. J. Princep, R. A. Ewings, S. Ward, S. Tóth, C. Dubs, D. Prabhakaran, and A. T. Boothroyd, npj Quantum Mater. 2, 63 (2017).

[59] S. Seki, T. Ideue, M. Kubota, Y. Kozuka, R. Takagi, M. Nakamura, Y. Kaneko, M. Kawasaki, and Y. Tokura, Phys. Rev. Lett. 115, 266601 (2015).

[60] S. M. Wu, W. Zhang, Amit KC, P. Borisov, J. E. Pearson, J. S. Jiang, D. Lederman, A. Hoffmann, and A. Bhattacharya, Phys. Rev. Lett. 116, 097204 (2016).

[61] V. Baltz, A. Manchon, M. Tsoi, T. Moriyama, T. Ono, and Y. Tserkovnyak, Rev. Mod. Phys. 90, 015005 (2018).

[62] J. L. Lado and M. Sigrist, Phys. Rev. Lett. 121, 037002 (2018). 